\title{
Association of SNPs in the PPAR $\gamma$ gene and hypertension in a Mongolian population
}

\author{
L. Yang, R.G. Tian, P.Y. Chang, M.R. Yan and X.L. Su \\ Clinical Medical Research Center of the Affiliated Hospital, \\ Inner Mongolia Medical University, Hohhot, China \\ Corresponding author: X.L. Su \\ E-mail: xlsu2015@163.com
}

Genet. Mol. Res. 14 (4): 19295-19308 (2015)

Received August 26, 2015

Accepted October 24, 2015

Published December 29, 2015

DOI http://dx.doi.org/10.4238/2015.December.29.39

ABSTRACT. The association of single nucleotide polymorphisms (SNPs) in PPAR $\gamma$ with hypertension is controversial. The aim of the present study was to clarify the contributions of PPAR $\gamma$ genetic variants to hypertension through an association study. A total of 414 unrelated Mongolian herdsmen and 524 Han farmers were included in this study. Fourteen intronic SNPs were analyzed and genotyped using a polymerase chain reaction/ligase detection reaction assay. Prior to correction for multiple testing, the SNPs rs6802898 and rs12633551 were significantly associated with the prevalence of hypertension in the Han and Mongolian populations, respectively. The genetic association of each SNP with hypertension was individually tested using logistic regression. The SNP rs6802898 was associated with hypertension in both dominant $(P=0.033)$ and additive models $(P=0.026)$ in the Han population, whereas the SNP rs12633551 was associated with hypertension in both dominant $(P=0.014)$ and additive models $(P=0.0073)$ in the Mongolian population. Moreover, SNP rs12633551 had a significant effect on systolic and diastolic blood pressure response. However, none of these associations were statistically significant after Bonferroni correction for multiple testing, although there was a significant difference among the haplotypes in the Han and Mongolian populations. Interestingly, there was an association of the PPAR $\gamma$ haplotypes with hypertension even after 
Bonferroni correction. Thus, determination of the PPAR $\gamma$ haplotypes in different populations may prove informative for assessment of the genetic risk for hypertension.

Key words: Essential hypertension; Haplotype; Mongolian population; PPAR $\gamma$ gene

\section{INTRODUCTION}

Hypertension is a major risk factor for developing ischemic heart disease, heart failure, cerebrovascular disease, atrial fibrillation, and chronic kidney disease (Benjamin et al., 1994; Kearney et al., 2004; Manolis et al., 2012). It has been shown to be the major cause of death over any other recognized risk factor globally. A report from the World Health Organization (WHO) indicated that, annually, 7.6 million premature deaths $(13.5 \%$ of the global total) are caused by elevated systolic blood pressure (SBP) levels of $115 \mathrm{mmHg}$ or above (Lawes et al., 2008).

Essential hypertension $(\mathrm{EH})$ is a complex trait and the underlying pathogenic mechanism is still poorly understood because of interplay between multiple genes and environmental factors. Familial studies showed a higher proportion of offspring with aggregation of blood pressure (BP) when parents had a history of hypertension with rates ranging from 20 to $66 \%$ in the general population (Harrap, 1994; Fuentes et al., 2000). In addition, the heritability estimates for EH from studies of monozygotic twins and dizygotic twins are greater than 50\% (Fagard et al., 1995; Somes et al., 1995). Although the heritability of hypertension has been established, the majority of the causative genes remain unknown.

Peroxisome proliferator-activated receptor $\gamma$ (PPAR $\gamma$ ) is a member of the steroid hormone receptor superfamily and is activated by a variety of chemical compounds and natural ligands (Desvergne and Wahli, 1999). It also has a well-recognized role in regulating multiple metabolic processes. PPAR $\gamma$ can regulate BP by modulation of the renin angiotensin aldosterone system (RAAS), which is an important pathway in managing systemic BP and interstitial fluid volume (Roszer and Ricote, 2010). In transgenic and knockout mice studies with mutant PPAR $\gamma$, it has been demonstrated that quantitative variants causing decreased PPAR $\gamma$ expression are potential contributors to EH (Halabi et al., 2008; Tsai et al., 2009). In humans, the role of PPAR $\gamma$ in BP regulation has been also revealed by genetic studies of patients with mutations in the PPAR $\gamma$ protein. However, it is still disputed whether the association of BP regulation and PPAR $\gamma$ mutations is dependent on insulin sensitivity (Deeb et al., 1998; Barroso et al., 1999; Ostgren et al., 2003).

A polymorphism in PPAR 2 (Pro12Ala) has been widely studied for its link to BP. Our previous study showed that the PPAR 2 Pro12Ala SNP is involved in genetic susceptibility to hypertension and metabolic lipid disorders in the Han population of Inner Mongolia (Gao et al., 2010). However, the association of PPAR 2 Pro12Ala with hypertension is controversial in other populations (Douglas et al., 2001; Ostgren et al., 2003; Rodriguez-Esparragon et al., 2003; Yliharsila et al., 2004; Gouni-Berthold et al., 2005; Sookoian et al., 2005). The analysis of haplotypes can provide additional information to investigate associations, particularly if the SNPs in the candidate gene are not causative. The aim of the present study was to clarify the contributions of PPAR $\gamma$ genetic variant haplotypes to hypertension through an association study. 


\section{MATERIAL AND METHODS}

\section{Subjects and measurements}

Subjects aged 20-75 years were enrolled from two villages in Duolun and three villages in Erenhot, Xilin Gol League, Inner Mongolia. Subjects with a history of secondary hypertension, stroke, coronary heart disease, diabetes, kidney failure, thyroid gland disease, or excessive drinking were excluded from this study. A total of 414 unrelated Mongolian herdsmen and 524 Han farmers were enrolled, including 177 Mongolian EH patients, 237 Mongolian normotensives (controls), 285 Han EH patients, and 239 Han normotensives (controls). However, 19 Mongolian EH patients did not undergo DNA genotyping. Each subject was from a family that had been living in Inner Mongolia for at least three generations without a history of mixed marriage. In 1999, the WHO defined hypertension as an SBP of at least $140 \mathrm{mmHg}$ and/or diastolic blood pressure (DBP) of at least $90 \mathrm{mmHg}$, or antihypertension treatment. This definition was adopted in this study. Individuals with secondary hypertension, diabetes mellitus, or severe liver, kidney and thyroid dysfunction were excluded from the study. The normotensive group was selected based on the following criteria: SBP of less than $140 \mathrm{mmHg}$, DBP of less than $90 \mathrm{mmHg}$, and no previous diagnosis of $\mathrm{EH}$. The subjects were seated in a quiet situation and prevented from smoking, exercising, or drinking alcohol, tea or coffee for at least $1 \mathrm{~h}$ before the physical examination. The following data were recorded for each subject: name, age, gender, nationality, height, weight, body mass index (BMI), history of alcohol and tobacco use, and BP (SBP and DBP). Smoking was defined as smoking at least one cigarette per day for at least 1 year, and alcohol consumption was defined as consuming $50 \mathrm{~g}$ or more of alcohol per day for at least 1 year. BP was measured three times, with a 2-min interval between each measurement. SBP was recorded to the nearest $2 \mathrm{mmHg}$ at the appearance of the first Korotkoff sound (phase I), and DBP was recorded to the nearest $2 \mathrm{mmHg}$ at the disappearance of the fifth Korotkoff sound (phase V). The SBP and DBP values were calculated as the means of three consecutive physician-obtained measurements. Body weight and height were measured with subjects wearing only light indoor clothing and no shoes. BMI was calculated by dividing weight $(\mathrm{kg})$ by height squared $\left(\mathrm{m}^{2}\right)$. Blood samples were collected after an overnight fast, and total plasma cholesterol (TC), triacylglycerides (TG), high-density lipoprotein cholesterol (HDL-C), and low-density lipoprotein cholesterol (LDL-C) were measured within $8 \mathrm{~h}$ of blood collection in a local hospital. Informed consent was obtained from all subjects. The protocol was reviewed and approved by the Ethics Committee of Affiliated Hospital of Inner Mongolia Medical College.

\section{DNA isolation and genotyping}

Tag SNPs were selected from the Chinese HapMap database (http://www.hapmap.org) using the Haploview program. Genomic DNA was extracted from peripheral blood leukocytes using a commercial blood DNA extraction kit (TaKaRa Biotechnology, Dalian, China) and was stored at $-20^{\circ} \mathrm{C}$. Fourteen tag SNPs of PPAR $\gamma$ were genotyped using the polymerase chain reaction $(P C R) / l i g a s e$ detection reaction assay. Primers were synthesized by Shanghai Sangon Biological Engineering Technology \& Services (China). Each set of ligase detection reaction probes comprised one common probe and two discriminating probes.

The target DNA sequences were amplified using a multiplex PCR method. PCR was carried out in a final volume of $10 \mu \mathrm{L}$, containing 1 X PCR buffer, $3 \mathrm{mM} \mathrm{MgCl}, 2 \mathrm{mM}$ deoxynucleotide triphosphate, $0.2 \mathrm{mM}$ each primer, $0.2 \mu \mathrm{L}$ Qiagen HotStarTaq Polymerase (QIAGEN, Shenzhen, 
China), $4 \mu \mathrm{L} 1 \mathrm{X}$ Q-solution, and 10-20 ng genomic DNA. Thermal cycling was performed for all SNP loci in a Gene Amp PCR system 9600 (PerkinElmer, Waltham, MA, USA) with the following program: an initial denaturation step at $95^{\circ} \mathrm{C}$ for $5 \mathrm{~min}$; 35 cycles of denaturation at $94^{\circ} \mathrm{C}$ for $40 \mathrm{~s}$, annealing at $55^{\circ} \mathrm{C}$ for $50 \mathrm{~s}$, and extension at $72^{\circ} \mathrm{C}$ for $1 \mathrm{~min}$; and final extension of $7 \mathrm{~min}$ at $72^{\circ} \mathrm{C}$. The ligation reactions were carried out in a final volume of $10 \mu \mathrm{L}$, containing 1 X NEB Taq DNA ligase buffer, 12.5 pmol each probe mix, $0.05 \mu \mathrm{L}$ Taq DNA ligase (NEB Biotechnology, Beijing, China), and $1 \mu \mathrm{L}$ multiPCR product. A total of 35 cycles of ligase detection reaction were performed with $95^{\circ} \mathrm{C}$ for 2 min, $94^{\circ} \mathrm{C}$ for $30 \mathrm{~s}$, and $60^{\circ} \mathrm{C}$ for $2 \mathrm{~min}$. The fluorescent products of the ligase detection reaction were differentiated by Applied Biosystems 3730 DNA Analyzer (Applied Biosystems, USA).

\section{Statistical analysis}

The quantitative variables were tested with the Student $t$-test or one-way analysis of variance. Categorical data were compared by the chi-square test $\left(\chi^{2}\right)$ or the Fisher test analysis. The allele frequencies, genotype frequencies, and Hardy-Weinberg equilibrium (HWE) were calculated for both the hypertension cases and controls by the chi-square test or the Fisher test analysis using SHEsis (Shi and $\mathrm{He}, 2005)$. For the haplotype analysis, the genotype data were used to measure the coefficient D' of linkage disequilibrium (LD) to construct haplotypes. Haplotypes with frequencies $>3 \%$ in the entire sample set were included in the association tests using the SHEsis software (Shi and He, 2005). The genetic association between the PPAR $\gamma$ polymorphisms and susceptibility to hypertension was individually tested by logistic regression with the following as covariates: gender, age, HDL level, TG level, TC level, LDL level, tobacco use, alcohol consumption, and BMI. For logistic regression, a webbased SNP analysis tool (Biostatistics and Bioinformatics Unit, Catalan Institute of Oncology, http:// bioinfo.iconcologia.net/ubbweb/SNPStats_web, was applied to assess each SNP genotype according to the three genetic models: additive, dominant, and recessive. ORs and $95 \% \mathrm{Cls}$ for hypertension were reported. The association of individual SNPs (additive, dominant, and recessive genetic models) with clinical characteristics was tested using the SPSS software (SPSS Inc., Chicago, IL, USA).

The effects of individual SNP genotypes on SBP and DBP of all the subjects were evaluated by general linear models adjusted with covariates (gender, ethnicity, tobacco use, alcohol consumption, HDL level, TG level, TC level, LDL level, BMI, height, and weight) using the SPSS software. The Bonferroni correction was used to adjust $P$ values for multiple measures as needed.

\section{RESULTS}

\section{Clinical characteristics and EH}

The clinical characteristics of hypertensive patients and controls in the Mongolian and Han populations are summarized in Table 1. Age, BMI, SBP, and DBP were significantly higher in hypertensive individuals than in the controls in both the Han and Mongolian populations. The gender distribution was similar between hypertensive individuals and controls in both populations. Subjects with hypertension had significantly lower levels of HDL-C compared with normotensive subjects in the Han population, whereas this difference was not observed in the Mongolian population. However, there were significant differences in LDL-C levels, TC levels, and alcohol consumption between hypertensive patients and controls in the Mongolian population. For TG levels and nicotine use (smoking), significant differences were observed between hypertensive patients and controls but with opposite trends in Mongolian and Han populations. The level of TG 
and proportion of subjects who smoked were higher in controls than in subjects with hypertension in the Han population. Conversely, the level of TG and proportion of subjects who smoked were higher in subjects with hypertension than controls in the Mongolian population.

Table 1. Characteristics of normotensive and hypertensive Mongolian and Han subjects.

\begin{tabular}{|c|c|c|c|c|c|c|}
\hline & \multicolumn{3}{|c|}{ Han } & \multicolumn{3}{|c|}{ Mongolian } \\
\hline & Controls ( $\mathrm{N}=239$ ) & Hypertension ( $\mathrm{N}=285$ ) & $P$ & Controls $(\mathrm{N}=237)$ & Hypertension $(\mathrm{N}=177)$ & $P$ \\
\hline Gender (Male/Female) & $155 / 84$ & $185 / 100$ & 0.989 & $98 / 139$ & $66 / 111$ & 0.403 \\
\hline Age (years) & $45.96 \pm 10.68$ & $49.85 \pm 11.92$ & $<0.001$ & $40.94 \pm 11.29$ & $51.53 \pm 11.50$ & $<0.0001$ \\
\hline BMI $\left(\mathrm{kg} / \mathrm{m}^{2}\right)$ & $23.89 \pm 3.31$ & $25.30 \pm 2.57$ & $<0.0001$ & $22.64 \pm 3.31$ & $24.32 \pm 2.99$ & $<0.0001$ \\
\hline $\mathrm{SBP}(\mathrm{mmHg})$ & $117.77 \pm 9.94$ & $146.77 \pm 18.03$ & $<0.0001$ & $108.57 \pm 12.41$ & $162.32 \pm 28.82$ & $<0.0001$ \\
\hline $\mathrm{DBP}(\mathrm{mmHg})$ & $76.21 \pm 8.17$ & $90.20 \pm 12.37$ & $<0.0001$ & $71.09 \pm 9.09$ & $104.41 \pm 14.41$ & $<0.0001$ \\
\hline HDL-C (mM) & $1.57 \pm 0.70$ & $1.20 \pm 0.41$ & $<0.0001$ & $1.98 \pm 1.42$ & $2.01 \pm 1.62$ & 0.851 \\
\hline LDL-C (mM) & $2.90 \pm 0.82$ & $3.01 \pm 0.94$ & 0.157 & $2.58 \pm 1.05$ & $3.59 \pm 1.32$ & $<0.0001$ \\
\hline TG $(\mathrm{mM})$ & $3.00 \pm 1.75$ & $2.09 \pm 1.59$ & 0.0001 & $1.58 \pm 0.91$ & $2.23 \pm 1.28$ & $<0.0001$ \\
\hline $\mathrm{TC}(\mathrm{mM})$ & $5.08 \pm 1.29$ & $4.94 \pm 0.96$ & 0.161 & $3.72 \pm 1.35$ & $5.31 \pm 1.95$ & $<0.0001$ \\
\hline Smoking (No/Yes) & $165 / 74$ & $219 / 66$ & 0.044 & $227 / 10$ & $142 / 35$ & $<0.0001$ \\
\hline Alcohol consumption (No/Yes & s) $\quad 156 / 83$ & $192 / 93$ & 0.613 & $201 / 36$ & $120 / 57$ & $<0.0001$ \\
\hline
\end{tabular}

Clinical characteristics of age, BMI, SBP, DBP, HDL-C, LDL-C, TG, and TC are given as means \pm SD and other values as number of individuals. $\mathrm{BMI}=$ body mass index; $\mathrm{SBP}=$ systolic blood pressure; $\mathrm{DBP}=$ diastolic blood pressure; $\mathrm{HDL}-\mathrm{C}=$ high-density lipoprotein cholesterol; TG = triglycerides; TC = total cholesterol; and LDL-C = low-density lipoprotein cholesterol.

\section{Genotype distributions}

The genotypic and allelic frequencies of all polymorphisms in the two separate populations and combined population complied with the Hardy-Weinberg equilibrium in both hypertensive patients and normotensive controls (data not shown). Comparison of genotype distributions between hypertensive individuals and controls by the $\chi^{2}$-test revealed that the presence of the rs6802898 and rs12633551 SNPs was significantly related to the prevalence of hypertension in the Han $(P=0.038)$ and Mongolian populations $(P=0.04)$, respectively (Table 2$)$. However, there was no difference in the genotype distribution and allelic frequency in the combined populations. SNPs rs6802898 $(P=0.011)$ and $r$ 13306745 $(P=0.019)$ had significantly different allelic frequencies between hypertensive individuals and controls in the Han population. SNP rs12633551 $(P=0.014)$ had significantly different allelic frequencies between hypertensive individuals and controls in the Mongolian population. However, when adjusted for multiple measures with a Bonferroni correction, none of the $P$ values $(P>0.05)$ were statistically significant in the Han and Mongolian populations.

\section{Genotypic association of SNPs with hypertension}

The genetic association of each SNP with hypertension was individually tested in dominant, recessive, and additive genetic models using logistic regression with hypertension and adjusted for gender, age, HDL level, TG level, TC level, LDL level, nicotine use, alcohol consumption, and BMI (Table 3). None of the SNPs had an effect on hypertension in the combined population. In the Han population, the rs6802898 SNP was associated with hypertension in both the dominant $(P=0.033)$ and additive models $(P=0.026)$, and the $\mathrm{rs} 12633551$ SNP was associated with hypertension in both the dominant $(P=0.014)$ and additive models $(P=0.0073)$ in the Mongolian population. However, following Bonferroni correction for multiple measures, no statistically significant associations were observed $(P>0.05)$. 


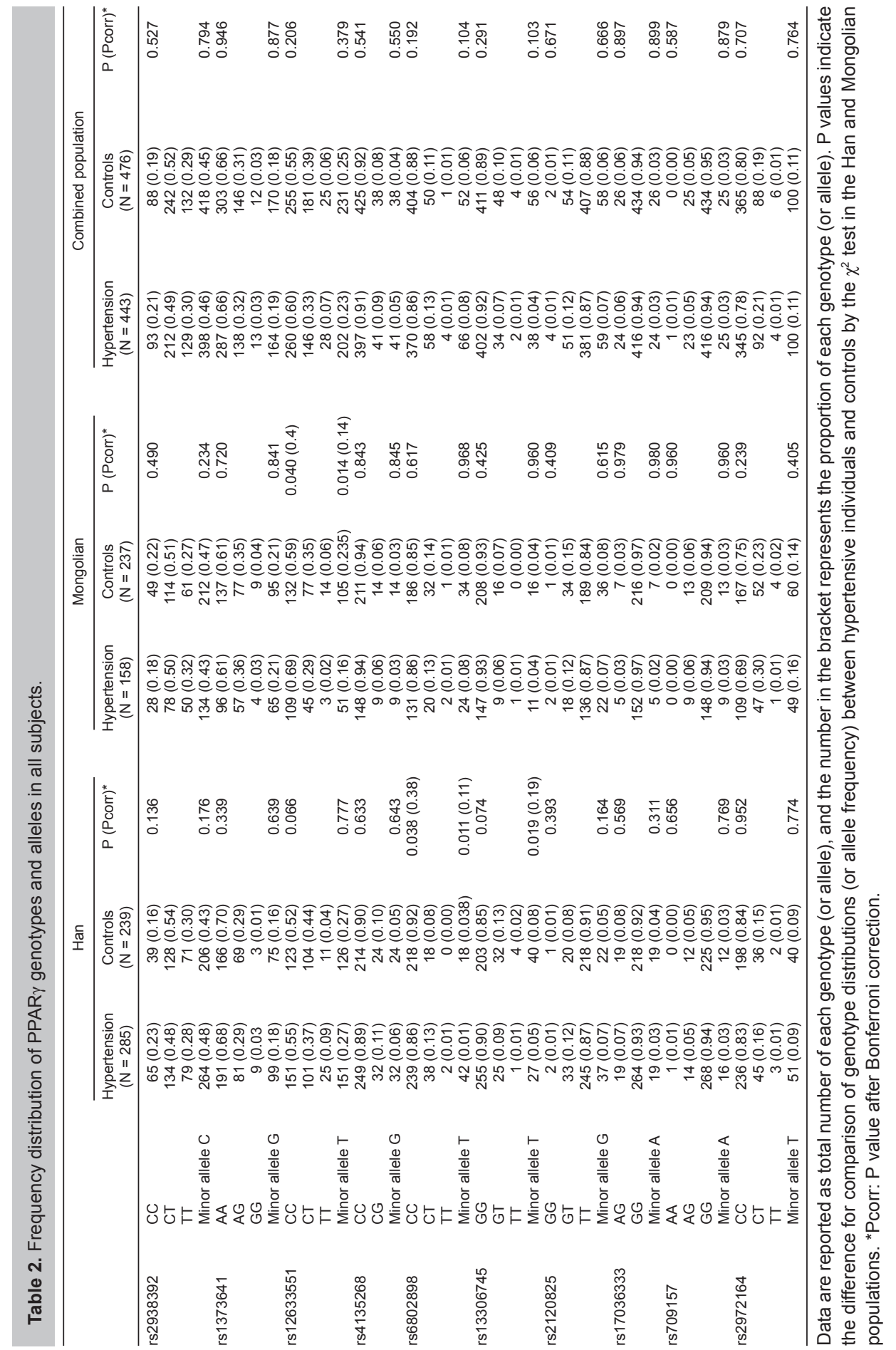




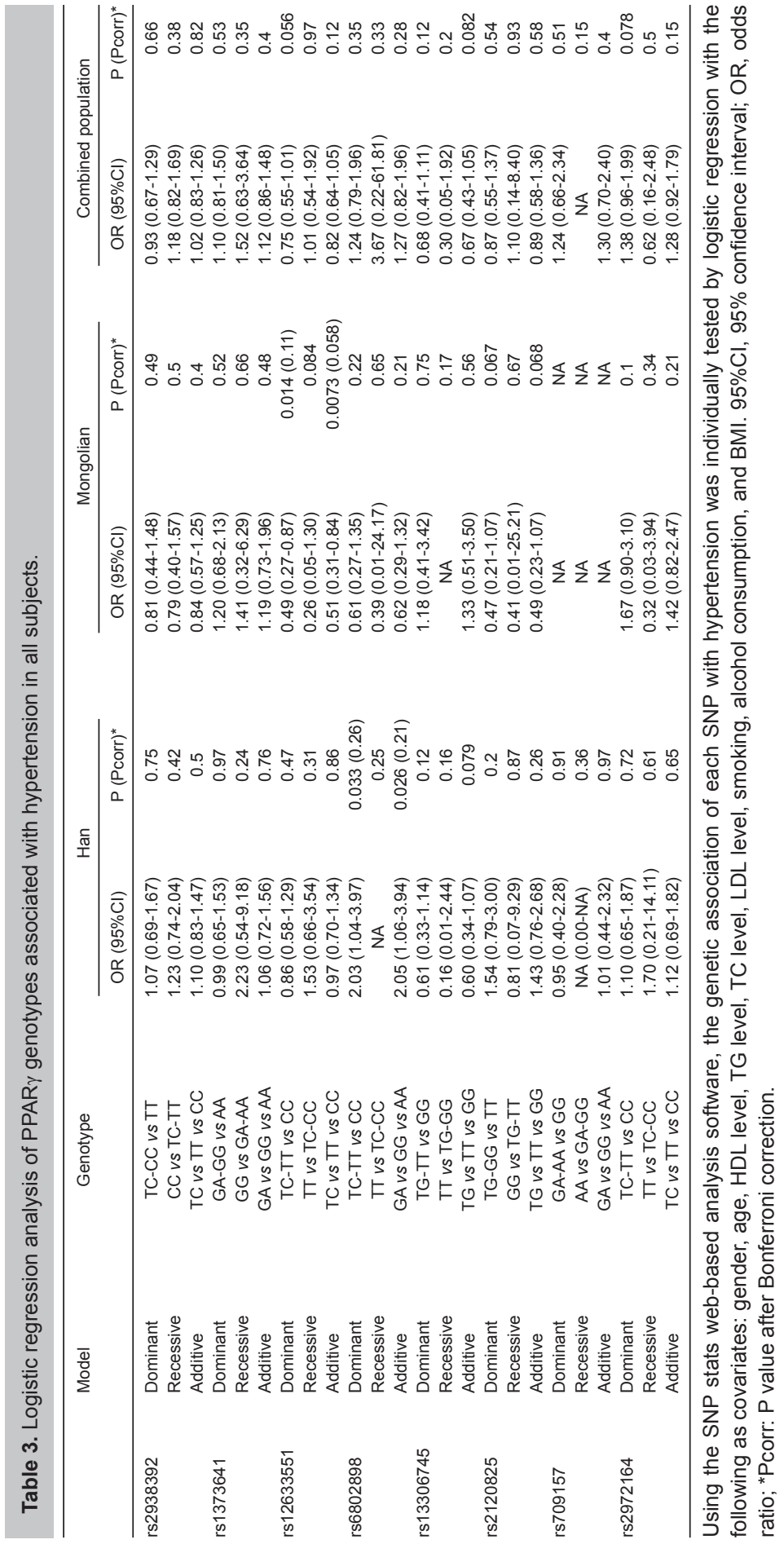




\section{Association of SNPs with BP}

The effects of the polymorphisms on systolic and diastolic BP were further examined in dominant, recessive, and additive models in the combined population (Table 4). Prior to correction for multiple testing, analysis revealed that only the rs12633551 SNP had a significant effect on BP. The genotype was significantly associated with DBP in the additive $(P=0.044)$ and dominant models $(P=$ 0.016). The effect on SBP was only significant in the dominant model $(P=0.036)$. However, none of these associations were statistically significant after Bonferroni correction $(P>0.05)$.

\section{Haplotype analysis}

The LD among the 14 PPAR $\gamma$ SNPs (rs2938392, rs4135325, rs796289, rs1373641, rs12633551, rs4135268, rs6802898, rs13306745, rs2120825, rs4135343, rs17036333, rs709157, rs2972164, and rs3773360) was examined in the Han and Mongolian populations. Both populations had similar patterns of LD (Figures S1 and $\underline{\mathbf{S 2}}$ ). The distribution of the haplotypes composed of these PPAR $\gamma$ SNPs (rs2938392, rs1373641, rs12633551, rs4135268, rs6802898, rs13306745, rs2120825, rs17036333, rs709157, and rs2972164) was analyzed with the SHEsis software. Since the minor allelic frequencies of SNPs rs4135325, rs796289, rs4135343, and rs3773360 were zero, they were excluded from the haplotype analysis. In addition, the haplotypes were excluded from further analysis if their frequencies were less than 0.03 in both the control and hypertension groups. There were eight and seven haplotypes with frequencies greater than 0.03 in both hypertensive patients and control groups in the Han and Mongolian populations, respectively (Table 5). In the global analysis, there was a significant difference between the hypertensive patient group and the control group in the Mongolian population $(P=0.02)$.

The "TACCCGTGGC" haplotype was the most frequent in the PPAR $\gamma$ gene in both populations. The frequency of the "TACCCGTGGC" haplotype was higher in the hypertensive patient group (0.33) than the control group (0.29) in the Mongolian population. In contrast, the frequency was higher in the control group (0.34) than the hypertensive patient group (0.31) in the Han population. Furthermore, in the Han population, the frequencies of the "TACCCTTGGC" and "TACCTGGGGC" haplotypes were significantly different between the hypertensive patient group and the control group $(P=0.02, O R=0.53,95 \% \mathrm{Cl}=0.30-0.92 ; \mathrm{P}=0.03, \mathrm{OR}=1.99$, $95 \% \mathrm{Cl}=1.06-3.76$, respectively). The frequencies of the "CATCCGTGGC" and "TACCCGTGGT" haplotypes exhibited a significant difference between the patient and the control groups $(P=$ $0.002, \mathrm{OR}=0.53,95 \% \mathrm{Cl}=0.35-0.79 ; \mathrm{P}=0.03, \mathrm{OR}=1.73,95 \% \mathrm{Cl}=1.04-2.85$, respectively) in the Mongolian population. However, after applying the stringent Bonferroni correction for multiple testing, only the "CATCCGTGGC" haplotype remained significantly associated with hypertension in the Mongolian population $(P=0.01)$.

\section{DISCUSSION}

PPAR $\gamma$ is located on chromosome 3p25. It is a ligand-activated transcription factor of the nuclear hormone receptor superfamily, and plays several important roles as the regulator of adipogenesis and glucose homeostasis. In addition, studies have provided strong evidence that PPAR $\gamma$ is involved in the regulation of vascular function and blood pressure (Barroso et al., 1999; Agostini et al., 2006; Keen et al., 2010). 
SNPs in the PPAR $\gamma$ gene and hypertension

19303

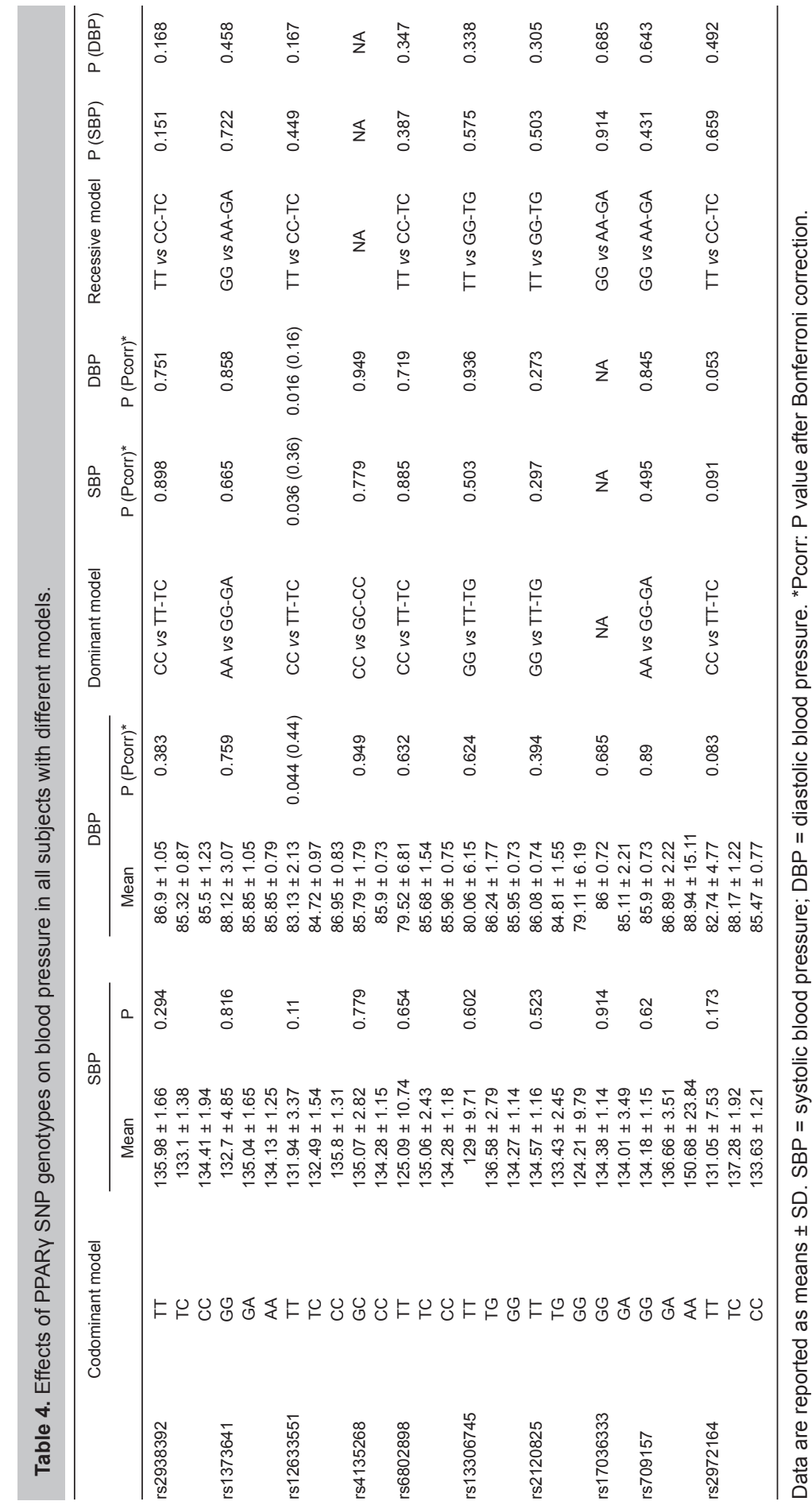




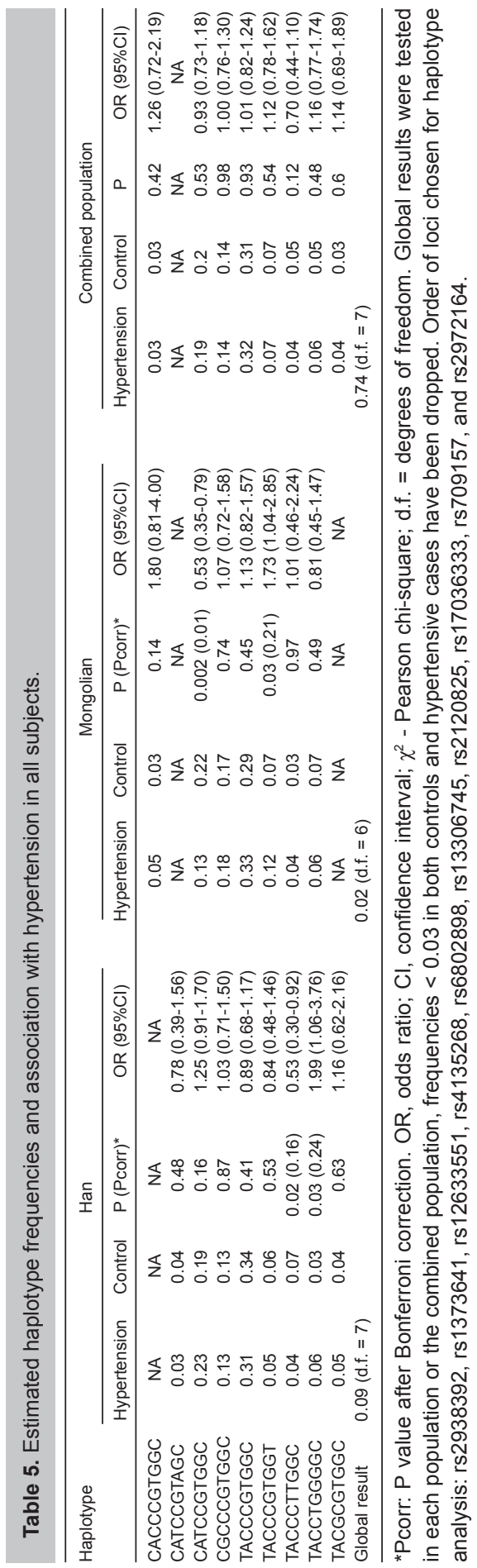


In this study, the association of intronic PPAR $\gamma$ SNPs with hypertension was tested in the Chinese Han and Mongolian populations, and the effects of the SNPs on the genetic contribution to hypertension were determined. Prior to correction for multiple testing, the rs6802898 and rs12633551 SNPs were identified as susceptible polymorphisms in Han and Mongolian hypertensive patients, respectively. Moreover, the rs12633551 SNP was found to be associated with BP in the combined population. However, the relationship between hypertension or BP and the SNPs was not statistically significant after applying the Bonferroni correction for multiple testing.

To our knowledge, the intronic polymorphisms included in this study have not been examined for their effects on hypertension. Most studies have focused on the coding polymorphisms in PPAR $\gamma$ in relation to hypertension. For example, the subjects with two mutations at codons 290 and 467 in PPAR $\gamma$ (Val290-to-Met and Pro467-to-Leu) were found to have elevated blood pressure, severe insulin resistance, and diabetes (Barroso et al., 1999). Individuals with a substitution in PPAR $\gamma$ at codon 388 (Phe388-to-Leu) (Hegele et al., 2002) or 425 (Arg425-to-Cys) (Agarwal and Garg, 2002) displayed both diabetic and hypertensive phenotypes. The Pro12-to-Ala mutation in PPAR $\gamma$ has been studied in depth. This polymorphism is significantly associated with hypertension, although its association with insulin sensitivity is still controversial (Deeb et al., 1998; Altshuler et al., 2000; Ostgren et al., 2003; Rodriguez-Esparragon et al., 2003; Yliharsila et al., 2004; Lu et al., 2008; Gao et al., 2010). The investigated polymorphisms herein are all located in intronic regions, which could play a role in regulating the expression level of a gene. These polymorphisms could be located within regulatory elements or microRNA coding sites, which result in gene expression differences, and thereby affect the pathogenesis of hypertension. Alternatively, it may be these SNPs are in LD with other functional polymorphisms in nearby regions.

The association of PPAR $\gamma$ polymorphisms with hypertension has been investigated in various populations (Douglas et al., 2001; Gouni-Berthold et al., 2005; Sookoian et al., 2005; Badii et al., 2008; Gallicchio et al., 2008; Dallongeville et al., 2009; Yoshida et al., 2009; Fan et al., 2010; Gao et al., 2010; Underwood et al., 2010; Shi et al., 2012). Given that the prevalence of hypertension is significantly different among races (Lackland et al., 2008), PPAR $\gamma$ was tested as a risk factor for hypertension in a Mongolian population. A previous study in a Mongolian population indicated that the prevalence of the Ala allele of the Pro12Ala polymorphism was significantly different between control and hypertension groups, which suggests a contribution of the PPAR $\gamma$ gene to hypertension susceptibility. However, there was no significant association between the Pro12Ala polymorphism and clinical and metabolic characteristics, including blood pressure. In the study herein, we provide further evidence that several intronic polymorphisms of the PPAR $\gamma$ gene are not associated with blood pressure. The association of two SNPs (rs6802898 and rs12633551) with hypertension was strongly dependent on ethnicity. SNP rs6802898 was only associated with susceptibility to hypertension in the Han Chinese population (allele frequency, $P=0.011$; genotype distribution, $P=0.038$ ). Furthermore, logistic regression analysis revealed that the association of this SNP with hypertension in a Han population was under the dominant $(P=0.033, O R=$ $2.03,95 \% \mathrm{Cl}=1.04-3.97)$ and additive $(\mathrm{P}=0.026, \mathrm{OR}=2.05,95 \% \mathrm{Cl}=1.06-3.94)$ models. SNP rs12633551 was the only susceptible variant in the Mongolian population (allele frequency, $\mathrm{P}=$ 0.014 ; genotype distribution, $\mathrm{P}=0.040)$, and the association with hypertension in the Mongolian population was dominant $(P=0.014, \mathrm{OR}=0.49,95 \% \mathrm{Cl}=0.27-0.87)$ and additive $(\mathrm{P}=0.0073$, $\mathrm{OR}=0.51,95 \% \mathrm{Cl}=0.31-0.84)$. Given the small sample size of subjects in the study herein, these associations may have occurred due to chance alone and may represent a false-positive result. Therefore, the analyses of the association of SNPs in the PPAR $\gamma$ gene with hypertension were corrected for the multiple tests. None of the associations for these SNPs were statistically significant 
after correction. However, applying the Bonferroni correction is overly conservative, reducing the ability to detect associations and resulting in an elevated false negative rate (type 2 error). Therefore, a more extensive study with a larger cohort is required in order to confirm the findings herein.

The analysis of haplotypes makes it possible to capture more of the underlying genetic variation than single SNPs alone. Fallin et al. (2001) provided evidence that the inheritance of haplotype combinations is often more effective for detecting associations than inheritance of a single SNP. The haplotype combination provides a more comprehensive approach of assessing the relationship between multisite variation and traits. Therefore, the analysis of haplotype combinations is more powerful than the analysis of single individual SNPs.

Haplotype analysis has been used to successfully localize the susceptibility genes related to hypertension in several studies (Fu et al., 2008; Ying et al., 2010). In the present study, the haplotypes composed of these PPAR $\gamma$ SNPs (rs2938392, rs1373641, rs12633551, rs4135268, rs6802898, rs13306745, rs2120825, rs17036333, rs709157, and rs2972164) had significant effects in the different populations. In the Han population, the frequency of the "TACCTGGGGC" haplotype in the hypertensive group was significantly higher than in the control group, indicating that this haplotype is associated with hypertension $(P=0.03, O R=1.99,95 \% \mathrm{Cl}=1.06-3.76)$. However, the same haplotype had no effect on hypertension in the Mongolian population $(P=$ 0.49). In contrast, two other haplotypes ("TACCCGTGGT" and "CATCCGTGGC") were associated with a higher prevalence of hypertension in the Mongolian population $(\mathrm{P}=0.03, \mathrm{OR}=1.73,95 \% \mathrm{Cl}$ $=1.04-2.85$ and $\mathrm{P}=0.002, \mathrm{OR}=0.53,95 \% \mathrm{Cl}=0.35-0.79$, respectively). It should be noted that the "CATCCGTGGC" haplotype remained significantly associated with hypertension even after applying the Bonferroni correction. Therefore, although the associations of each SNP were not always significant, the haplotypes formed from PPAR $\gamma$ SNPs still had effects on susceptibility to hypertension but were ethnicity-dependent.

In conclusion, this study showed that PPAR $\gamma$ may be a susceptibility gene for hypertension in the Mongolian and Han populations. It was notable that the association of the PPAR $\gamma$ polymorphisms and their haplotypes with hypertension is related to ethnicity. Determination of the genotype of PPAR $\gamma$ polymorphisms and their haplotypes in different populations may prove informative for assessment of the genetic risk for hypertension. Due to the small population size used herein, validation with another independent set of subjects is necessary to confirm these findings.

\section{Conflicts of interest}

The authors declare no conflict of interest.

\section{ACKNOWLEDGMENTS}

Research supported by the National Natural Science Foundation of China (\#81260058) and the Inner Mongolia Natural Science Foundation Key Program (\#2010Zd26).

\section{Supplementary material}

\section{REFERENCES}

Agarwal AK and Garg A (2002). A novel heterozygous mutation in peroxisome proliferator-activated receptor-gamma gene in a patient with familial partial lipodystrophy. J. Clin. Endocrinol. Metab. 87: 408-411. 
Agostini M, Schoenmakers E, Mitchell C, Szatmari I, et al. (2006). Non-DNA binding, dominant-negative, human PPAR gamma mutations cause lipodystrophic insulin resistance. Cell Metab. 4: 303-311.

Altshuler D, Hirschhorn JN, Klannemark M, Lindgren CM, et al. (2000). The common PPARgamma Pro12Ala polymorphism is associated with decreased risk of type 2 diabetes. Nat. Genet. 26: 76-80.

Badii R, Bener A, Zirie M, Al-Rikabi A, et al. (2008). Lack of association between the Pro(12) Ala polymorphism of the PPARgamma 2 gene and type 2 diabetes mellitus in the Qatari consanguineous population. Acta Diabetol. 45: $15-21$.

Barroso I, Gurnell M, Crowley VEF, Agostini M, et al. (1999). Dominant negative mutations in human PPARgamma associated with severe insulin resistance, diabetes mellitus and hypertension. Nature 402: 880-883.

Benjamin EJ, Levy D, Vaziri SM, Dagostino RB, et al. (1994). Independent risk factors for atrial fibrillation in a population-based cohort. The Framingham Heart Study. JAMA 271: 840-844.

Dallongeville J, Iribarren C, Ferrieres J, Lyon L, et al. (2009). Peroxisome proliferator-activated receptor gamma polymorphisms and coronary heart disease. PPAR Res. 2009: 543746.

Deeb SS, Fajas L, Nemoto M, Pihlajamaki J, et al. (1998). A Pro12Ala substitution in PPAR gamma 2 associated with decreased receptor activity, lower body mass index and improved insulin sensitivity. Nat. Genet. 20: 284-287.

Desvergne B and Wahli W (1999). Peroxisome proliferator-activated receptors: nuclear control of metabolism. Endocr. Rev. 20: $649-688$.

Douglas JA, Erdos MR, Watanabe RM, Braun A, et al. (2001). The peroxisome poliferator-activated receptor-gamma 2 Pro12Ala variant: association with type 2 diabetes and trait differences. Diabetes 50: 886-890.

Fagard R, Brguljan J, Staessen J, Thijs L, et al. (1995). Heritability of conventional and ambulatory blood pressures. A study in twins. Hypertension 26: 919-924.

Fallin D, Cohen A, Essioux L, Chumakov I, et al. (2001). Genetic analysis of case/control data using estimated haplotype frequencies: application to APOE locus variation and Alzheimer's disease. Genome Res. 11: 143-151.

Fan AZ, Yesupriya A, Chang MH, House M, et al. (2010). Gene polymorphisms in association with emerging cardiovascular risk markers in adult women. BMC Med. Genet. 11: 6.

Fu Z, Nakayama T, Sato N, Izumi Y, et al. (2008). Haplotype-based case-control study of the human CYP4F2 gene and essential hypertension in Japanese subjects. Hypertens. Res. 31: 1719-1726.

Fuentes RM, Notkola IL, Shemeikka S, Tuomilehto J, et al. (2000). Familial aggregation of blood pressure: a population-based family study in eastern Finland. J. Hum. Hypertens. 14: 441-445.

Gallicchio L, Kalesan B, Huang H-Y, Strickland P, et al. (2008). Genetic polymorphisms of peroxisome proliferator-activated receptors and the risk of cardiovascular morbidity and mortality in a community-based cohort in Washington County, Maryland. PPAR Res. 2008: 276581.

Gao L, Wang L, Yun H, Su L, et al. (2010). Association of the PPAR gamma 2 gene Pro12Ala variant with primary hypertension and metabolic lipid disorders in Han Chinese of Inner Mongolia. Genet. Mol. Res. 9: 1312-1320.

Gouni-Berthold I, Giannakidou E, Muller-Wieland D, Faust M, et al. (2005). Peroxisorne proliferator-activated receptor-gamma 2 Pro12Ala and endothelial nitric oxide synthase-4a/b gene polymorphisms are not associated with hypertension in diabetes mellitus type 2. J. Hypertens. 23: 301-308.

Halabi CM, Beyer AM, De Lange WJ, Keen HL, et al. (2008). Interference with PPAR gamma function in smooth muscle causes vascular dysfunction and hypertension. Cell Metab. 7: 215-226.

Harrap SB (1994). Hypertension: genes versus environment. Lancet 344: 169-171.

Hegele RA, Cao HN, Frankowski C, Mathews ST, et al. (2002). PPARG F388L, a transactivation-deficient mutant, in familial partial lipodystrophy. Diabetes 51: 3586-3590.

Kearney PM, Whelton M, Reynolds K, Whelton PK, et al. (2004). Worldwide prevalence of hypertension: a systematic review. J. Hypertens. 22: 11-19.

Keen HL, Halabi CM, Beyer AM, de Lange WJ, et al. (2010). Bioinformatic analysis of gene sets regulated by ligand-activated and dominant-negative peroxisome proliferator-activated receptor gamma in mouse aorta. Arterioscler. Thromb. Vasc. Biol. 30: 518-525.

Lackland DT, Egan BM, Mountford WK, Boan AD, et al. (2008). Thirty-year survival for black and white hypertensive individuals in the Evans County Heart Study and the Hypertension Detection and Follow-up Program. J. Am. Soc. Hypertens. 2: 448-454.

Lawes CM, Vander Hoorn S and Rodgers A (2008). Global burden of blood-pressure-related disease, 2001. Lancet 371: $1513-1518$.

Lu ZC, Dong BR, Mo XM, Chen T, et al. (2008). Pro12Ala polymorphism in PPAR gamma 2 associated with essential hypertension in Chinese nonagenarians/centenarians. Exp. Gerontol. 43: 1108-1113.

Manolis AJ, Rosei EA, Coca A, Cifkova R, et al. (2012). Hypertension and atrial fibrillation: diagnostic approach, prevention and treatment. Position paper of the Working Group 'Hypertension Arrhythmias and Thrombosis' of the European Society of Hypertension. J. Hypertens. 30: 239-252.

Ostgren CJ, Lindblad U, Melander O, Melander A, et al. (2003). Peroxisome proliferator-activated receptor-gamma Pro12Ala polymorphism and the association with blood pressure in type 2 diabetes: Skaraborg Hypertension and Diabetes Project. J. Hypertens. 21: 1657-1662. 
Rodriguez-Esparragon FJ, Rodriguez-Perez JC, Macias-Reyes A and Alamo-Santana F (2003). Peroxisome proliferatoractivated receptor-gamma2-Pro12Ala and endothelial nitric oxide synthase-4a/b gene polymorphisms are associated with essential hypertension. J. Hypertens. 21: 1649-1655.

Roszer T and Ricote M (2010). PPARs in the renal regulation of systemic blood pressure. PPAR Res. 2010: 698730.

Shi H, Lu Y, Du J, Du WC, et al. (2012). Application of back propagation artificial neural network on genetic variants in adiponectin $A D I P O Q$, peroxisome proliferator-activated receptor-gamma, and retinoid $\mathrm{X}$ receptor-alpha genes and type 2 diabetes risk in a Chinese Han population. Diabetes Technol. Ther. 14: 293-300.

Shi YY and He L (2005). SHEsis, a powerful software platform for analyses of linkage disequilibrium, haplotype construction, and genetic association at polymorphism loci. Cell Res. 15: 97-98.

Somes GW, Harshfield GA, Alpert BS, Goble MM, et al. (1995). Genetic influences on ambulatory blood pressure patterns. The Medical College of Virginia Twin Study. Am. J. Hypertens. 8: 474-478.

Sookoian S, Garcia SI, Porto PI, Dieuzeide G, et al. (2005). Peroxisome proliferator-activated receptor gamma and its coactivator-1 alpha may be associated with features of the metabolic syndrome in adolescents. J. Mol. Endocrinol. 35: 373-380.

Tsai YS, Xu L, Smithies O and Maeda N (2009). Genetic variations in peroxisome proliferator-activated receptor y expression affect blood pressure. Proc. Natl. Acad. Sci. U.S.A. 106: 19084-19089.

Underwood PC, Sun B, Williams JS, Pojoga LH, et al. (2010). The relationship between peroxisome proliferator-activated receptor-y and renin: a human genetics study. J. Clin. Endocrinol. Metab. 95: E75-E79.

Ying CQ, Wang YH, Wu ZL, Fang MW, et al. (2010). Association of the renin gene polymorphism, three angiotensinogen gene polymorphisms and the haplotypes with essential hypertension in the mongolian population. Clin. Exp. Hypertens. 32: 293-300.

Yliharsila H, Eriksson JG, Forsen T, Laakso M, et al. (2004). Interactions between peroxisome proliferator-activated receptorgamma 2 gene polymorphisms and size at birth on blood pressure and the use of antihypertensive medication. $J$. Hypertens. 22: 1283-1287.

Yoshida T, Kato K, Yokoi K, Watanabe S, et al. (2009). Association of candidate gene polymorphisms with chronic kidney disease in Japanese individuals with hypertension. Hypertens. Res. 32: 411-418. 